\title{
Optimal Sizing of Battery Energy Storage System in a Shipboard Power System with considering Energy Management Optimization
}

\author{
Xianqiang Bao, ${ }^{1}$ Xinghua $X u^{1}{ }^{1}$ Yan $Z_{\text {hang }}{ }^{D},{ }^{1}$ Yiyong Xiong, ${ }^{2}$ and Chengya Shang ${ }^{1}$ \\ ${ }^{1}$ National Key Laboratory of Science and Technology on Vessel Integrated Power System, Naval University of Engineering, \\ Wuhan, China \\ ${ }^{2}$ College of Electrical Engineering, Naval University of Engineering, Wuhan, China \\ Correspondence should be addressed to Yan Zhang; zy331214534@126.com
}

Received 7 May 2021; Revised 28 June 2021; Accepted 2 July 2021; Published 13 July 2021

Academic Editor: Shi Cheng

Copyright (C) 2021 Xianqiang Bao et al. This is an open access article distributed under the Creative Commons Attribution License, which permits unrestricted use, distribution, and reproduction in any medium, provided the original work is properly cited.

Due to the increasing concerns about the environmental and economic issues of traditional ships, all-electric ships with energy storage and renewable energy integration have become more and more appealing for the forthcoming future. In this paper, an optimal energy storage system (ESS) capacity determination method for a marine ferry ship is proposed; this ship has diesel generators and PV panels. ESSs sizing optimization and power system scheduling optimization are simultaneously conducted and it is converted to a mixed-integer quadratic programming (MIQP) model with special modeling techniques. The case study shows that the proposed method is flexible and effective, and the relationships between the ESSs size and the discharge rate, life cycle times, or initial investment cost are investigated.

\section{Introduction}

The impacts of global warming caused by air pollution and depletion of fossil fuels have attracted increasing attention and opportunities in transportation, especially in the maritime industry. According to the International Maritime Organization (IMO) reports, maritime transportation contributes to $2.2 \%$ of $\mathrm{CO}_{2}$ emissions in the world in 2012, and if no action is taken, this data will increase between $50 \%$ and $250 \%$ in 2050 [1]. Therefore, the IMO requires the shipbuilding industry to improve the efficiency of the onboard energy systems for the mitigation of $\mathrm{CO}_{2}$ emissions [2-4]. In this context, the integrated power system (IPS) technology is used for the all-electric ship (AES), which combines electric propulsion and ship service electric grid to provide a common electrical platform [5] has become an appealing technology compared to the traditional ships. Since its superior energy efficiency, IPS technology is attracting more and more attention [6].

A few years ago, solar photovoltaic has aroused a high degree of interest in the scientific community and has been involved in some experimental research; however, the high expensive PV investment cost prevents its widespread use in ship area [7]. Recently, due to the oil price increases and PV investment costs decreasing, more and more ships choose to deploy a few PV panels on the rooftop to reduce the ship's greenhouse gas emissions, improve energy efficiency, and save operation costs.

\section{Literature Review}

2.1. Literature Survey. In a ship with IPS, the variable frequency drive technology, unit commitment technology $[8,9]$, and demand-side management algorithm can be used to improve system efficiency and fuel economy. However, as the most important load, the propulsion power is significantly impacted by the uncertain ocean and weather conditions, which causes the outputs of the generators to vary greatly, which then further makes the advantages of the AES not fully displayed.

Motivated by the successful application experience of energy storage systems (ESSs) in mitigating the negative impacts introduced by the uncertainties of renewable energy resources $[10,11]$, the importance of onboard ESSs and the 
smart energy management strategies for shipboard microgrid has been discussed in [12]. ESS can absorb energy from the ship power system when the load is low and release it when the load becomes high, which can reduce the fuel consumption and improve the economy of the IPS.

Although ESSs have so many advantages, their expensive investment cost and the limited ship deployment space prevent them from widely applying. In this situation, it is necessary to address the problem of ESS sizing by considering the optimal power scheduling problem, which has a close relationship to the performance of ESS $[13,14]$.

Lots of recent research works have been done in the area of optimal system designation and ESS sizing on the land grid [15]. References [16, 17] proposed a rule-based ESS size optimization approach; the ESS charging/discharging power is obtained by implementing the discrete Fourier transform (DFT) algorithm to the system imbalance power. In [18], ESS capacity optimization models based on rule operation strategy and optimal operation strategy are considered and discussed. Simulation results show that, compared with the traditional rule-based ESS size optimization approaches, the optimal operation strategy-based ESS capacity optimization model can significantly reduce battery capacity degradation and microgrid operation cost. To solve the ESS capacity optimization problem, some coevolutionary algorithms [19], deep learning algorithms [20], and meta-heuristic algorithms in land microgrids or large power system are used. Although these algorithms have superior modeling construction and searching the optimal objectives, however, the computation burden of these algorithms will be very large if the ESS operation strategy is considered in the ESS sizing optimization problems and a long time will be used to obtain the final result.

Due to the shipboard ESS sizing problem with optimal energy management strategy involving too many variables, parameters, and specific constraints, some studies [21-23] proposed the two-stage modeling method. They divided the whole optimization problem into two sub-problems. The first one is the traditional optimal sizing problem, which is used to determine the ESS capacity and solved by the heuristic or meta-heuristics approaches [24, 25], and this sub-problem does not consider the detailed system operation plan. While the second one is the traditional optimal energy management problem, its ESS parameters are obtained from the result of the first sub-problem and the results of the second sub-problem are the operation schemes of the DGs and the ESS units. However, this modeling and solving approach has to take a long time to obtain the optimal ESS size because the two sub-problems should be iterated lots of times to choose the minimal value. Literature of [26] proposed a hybrid modeling method of ESS optimization for a ferry, an SOC based ESS optimization is considered as the inner control loop, and the ESS sizing optimization is considered as the outer loop; the final result is obtained by combining the two-loop results. Reference [27] proposed a comprehensive modeling and optimization technology, which can integrate the ESS capacity optimization problem and optimal energy management problem into a single model. However, the optimal energy management model used is too simple to lose many important features of the ESS and DGs, such as the charging/discharging efficiency, the power ramp rate, and the minimum run/downtime.

2.2. Contributions. This work is focused on proposing a new modeling approach to find the best ESS size of a ship power system with considering the optimal energy management strategy. The most motivation is to simultaneously optimize the ESS sizing problem and the optimal energy management problem in a mathematical model. Due to the unit commitment problem, ESS optimal schedule, demand-side management, and sizing problem are included; this mathematical model is expressed by a mixed-integer quadratic programming (MIQP) model.

2.3. Organization. The outline of this paper is as follows: Section 3 introduces the structure of the shipboard IPS and the operation features of ESS and DGs. Section 4 describes the detailed modeling process of the ship power system ESS sizing problem. Results and discussions are implemented in Section 5, and Section 6 concludes.

\section{Problem Description}

This section describes the framework of IPS, PV generation model, DG efficient operation characteristics, and the life model of the ESS.

3.1. Integrated Power System (IPS) Description. A generic diagram of an IPS is shown in Figure 1; it can be considered as an independent shipboard DC microgrid. Compared with the traditional ships, the generators in the IPS supply power to the ship service load and electric propulsion motors simultaneously. Therefore, the power of these two parts can be adjusted at any time through the energy management system (EMS) to increase the fuel efficiency or improve the power quality [28]. In the IPS, the prime movers can be gas turbines or diesel generators, or both of them. Besides, the generators are all rectification generators.

To guarantee the ship power system safety, the IPS operation should be subject to several constraints and limitations, such as generation and consumption balance limitation, ship speed variation limitation, spinning reserve limitation, and generator ramp rate limitation. Besides, the gas emission limitation should also be contained in the near future.

3.2. PV Generation. As we all know, the power output of PV panels is determined by many factors, such as the effective solar radiation on the panels, the $\mathrm{PV}$ panel type, and the PV panel slope angle $[29,30]$. Mathematically, the PV generation can be described as the following. 


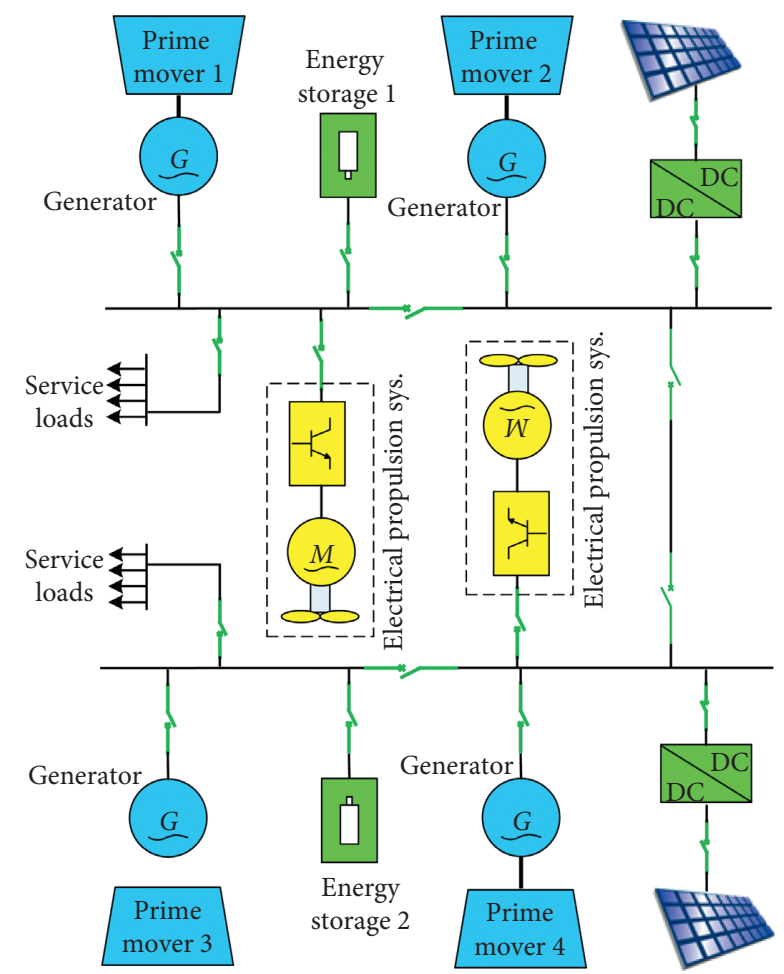

FIGURE 1: Generic diagram of IPS.

$$
\begin{aligned}
T_{C}(t) & =T_{A}(t)+\frac{\mathrm{NCOT}-20}{800} S_{p}(t, \beta), \\
I_{\mathrm{SC}}(t, \beta) & =\left[I_{\mathrm{SC}, \mathrm{STC}}+K_{I}\left(T_{C}(t)-25\right)\right] \frac{S_{p}(t, \beta)}{1000}, \\
V_{\mathrm{OC}}(t) & =V_{\mathrm{OC}, \mathrm{STC}}-K_{V} T_{C}(t), \\
P_{\mathrm{PV}}(t) & =N_{\mathrm{PV}} V_{\mathrm{OC}}(t) I_{\mathrm{SC}}(t, \beta) \mathrm{FF}(t),
\end{aligned}
$$

where $T_{C}(t)$ and $T_{A}(t)$ are the PV panel temperature and ambient temperature, respectively. NCOT is short for Nominal Cell Operating Temperature; its value is generally provided by the manufacturer. $I_{\mathrm{SC}, \mathrm{STC}}$ and $V_{\mathrm{OC}, \mathrm{STC}}$ are the short-circuit current and open-circuit voltage of the PV panel under the Standard Test Conditions; namely, the ambient temperature is $25^{\circ} \mathrm{C}$ and the effective solar radiation on the panels is $1 \mathrm{~kW} / \mathrm{m}^{2}, K_{I}$ and $K_{V}$ are the corresponding temperature coefficients, $P_{\mathrm{PV}}(t)$ is the $\mathrm{PV}$ generation with $N_{\mathrm{PV}} \mathrm{PV}$ panels, $\mathrm{FF}(t)$ is a fill factor which is determined by the type of PV panels, $\beta$ is the slope angle of the PV panel, and $S_{p}(t, \beta)$ is the effective solar radiation for the PV panel, which is described as the following:

$$
S_{p}(t, \beta)=\frac{S}{\sinh } \sin (h+\beta)
$$

$$
\sinh =\sin \varphi \sin \delta+\cos \varphi \cos \delta \cos \tau,
$$

where $h$ is solar elevation, $\varphi$ is geography of the latitude, $\tau$ is hour angle, and $\delta$ is solar declination angle.
3.3. Efficient Operation of Diesel Generators. Generally, the fuel consumption cost of the ship diesel generator $C_{i, \mathrm{DG}}^{\text {fuel }}$ can be approximated by a second-order polynomial function:

$$
C_{i, \mathrm{DG}}^{\text {fuel }}\left(P_{i, \mathrm{DG}}\right)=\alpha_{i} P_{i, \mathrm{DG}}^{2}+\beta_{i} P_{i, \mathrm{DG}}+\gamma_{i} \delta_{i, \mathrm{DG}}^{\text {onoff }},
$$

where $P_{i, \mathrm{DG}}(t)$ is power output of the $i^{\text {th }} \mathrm{DG}$ in period $t$ $(\mathrm{kW}), \delta_{i, \mathrm{DG}}^{\text {onoff }}$ is operation state of the $i^{\text {th }} \mathrm{DG}$ in period $t$ (binary), and $\alpha_{i}, \beta_{i}, \gamma_{i}$ are cost coefficients of the $i^{\text {th }} \mathrm{DG}$ $\left(\$ / \mathrm{MWh}^{2}, \$ / \mathrm{MWh}, \$ / \mathrm{h}\right)$.

Specific fuel consumption (SFC) is the result of fuel consumption cost divide by power output, which is expressed as equation (4). It is used to realize the operation characteristic of the generators.

$$
\operatorname{SFC}_{i, \mathrm{DG}}^{\mathrm{fuel}}\left(P_{i, \mathrm{DG}}\right)=\alpha_{i} P_{i, \mathrm{DG}}+\beta_{i}+\frac{\gamma_{i}}{P_{i, \mathrm{DG}}}
$$

Equation (4) indicates that once a generator is started, its SFC first decreases when its power output is smaller than the most efficient power point; then the SFC will increase continuously.

The $\mathrm{CO}_{2}$ emission is determined by the power output of the DGs. It can be expressed as

$$
E_{i, \mathrm{DG}}^{\text {fuel }}\left(P_{i, \mathrm{DG}}\right)=a_{i} P_{i, \mathrm{DG}}^{2}+b_{i} P_{i, \mathrm{DG}}+c_{i} \delta_{i, \mathrm{DG}}^{\text {onoff }},
$$

where $a_{i}, b_{i}, c_{i}$ are $\mathrm{CO}_{2}$ emission of the $i^{\text {th }} \mathrm{DG}\left(\mathrm{kg} / \mathrm{kW}^{2}, \mathrm{~kg} /\right.$ $\mathrm{kW}, \mathrm{kg} / \mathrm{h}$ ).

3.4. ESS Efficient Operation. There are several types of ESSs available on the market, in which the battery due to its high specific energy and suitable specific power becomes the most widely used ESS $[31,32]$. However, one of the shortcomings of the battery is its short life cycle which leads to the whole life cost of the battery being a little expensive.

According to the literature, the battery life is mainly affected by the depth of discharge (DOD) and the number of charge-discharge cycles [33]. The degradation of batteries in each charge-discharge cycle can be modeled as [34]:

$$
N_{\text {cycle }}=a \cdot e^{b \cdot \mathrm{DoD}_{\text {avg }}}+c \cdot e^{d \cdot \mathrm{DoD}_{\text {avg }},}
$$

where $N_{\text {cycle }}$ is the number of charge-discharge cycles to a fault, $\mathrm{DoD}_{\mathrm{avg}}$ is the preset DOD value which should be subjected over its life, $a, b, c, d$ are the fitting parameters, they are constants and provided by the manufacture, and their values are determined by the value of $\mathrm{DoD}_{\text {avg }}$. After using $N_{\text {cycle }}$ cycles, the battery is assumed to have reached the end of its life and should be replaced.

For most batteries, the manufacturer will provide the suggestion of how to set the $\mathrm{DoD}_{\mathrm{avg}}$ value to achieve the best life according to their long-term user experience.

\section{Materials and Methods}

This section provides the modeling process of how to calculate the best ESS capacity. 
4.1. Objective Function. The goal is to find the optimal ESS size, which can make the total value of the IPS operation cost $C_{M}$ and the ESS investment cost $C_{I}$ over the ship life be the minimal.

$$
\min C_{\text {total }}=\min \left(C_{I}+C_{M}\right) .
$$

Due to $C_{\text {total }}$ including investment cost and operation cost, its minimum value must be the optimized result both considered the ESS size and operation schedule.

4.1.1. ESS Investment Cost. The investment cost $C_{I}$ equals the summary of the storage pack initial investment cost $C_{I_{-} \text {ESS }}$, converter initial investment cost $C_{I_{-} \text {inverter }}$ and storage pack replacement cost $C_{R}[10]$.

$$
C_{I}=C_{I_{-} \mathrm{ESS}}+C_{I_{-} \text {inverter }}+C_{R} .
$$

In equation (8), the storage pack initial investment cost $C_{I_{-} \text {ESS }}$ is expressed as the product of the single module cost $C_{n \mathrm{ESS}}$, the module nominal capacity $E_{\mathrm{ESS}}^{\mathrm{max}}$, and the number of modules $N_{\text {ESS }}$. $N_{\text {ESS }}$ is one of the determination variables, which determines the ESS size.

$$
C_{I_{-} \mathrm{ESS}}=C_{n \mathrm{ESS}} \cdot E_{n \mathrm{ESS}}^{\mathrm{max}} \cdot N_{\mathrm{ESS}} .
$$

In this paper, we use the module as the minimal component of storage pack to reduce the value of the decision variable. A storage module consists of several storage cells.

Similar to the initial investment cost of storage pack, the initial investment cost of the converter can be expressed as the product of every kilowatt cost $C_{n i n v e r t e r}$ and the rated ESS power:

$$
C_{I_{-} \text {inverter }}=C_{\text {ninverter }} \cdot P_{\mathrm{ESSd}}^{\max } \cdot N_{\mathrm{ESS}},
$$

where $P_{\mathrm{ESSd}}^{\max }$ is the rated discharge power of a storage module. It is equal to the product of its nominal capacity and the discharge rate

$$
P_{\mathrm{ESSd}}^{\max }=E_{n \mathrm{ESS}}^{\max } \cdot \mathrm{RD}_{n \mathrm{ESS}} .
$$

Due to the feature limitation of some ESS technologies, for example, the charge rate $\mathrm{RC}_{n \mathrm{ESS}}$ of the battery is different from the discharge rate $\mathrm{RD}_{n \mathrm{ESS}}$, which results in its rated discharge and charge powers are different. The rated charge power of a storage module isz

$$
P_{\mathrm{ESSc}}^{\max }=E_{n \mathrm{ESS}}^{\max } \cdot \mathrm{RC}_{n \mathrm{ESS}} .
$$

Since the life cycle of ESS converter is very long, the replacement action can be neglected over the ship life. However, the higher energy density ESS usually has a shorter life. Therefore, the replacement operation of ESS pack should be considered [35]. The replacement cost $C_{R}$ for the storage pack is illustrated in the following equation:

$$
C_{R}=C_{I_{-} \mathrm{ESS}} \cdot N_{\text {repESS}} .
$$

Equation (13) indicates that $C_{R}$ is directly determined by the number of storage replacement $N_{\text {repESS }}$ [15], and the formulation of $N_{\text {repESS }}$ is expressed as follows:

$$
N_{\text {repESS }}=\frac{\left(T_{\text {ship }} \cdot 365\right)}{N_{\text {use_days }}},
$$

where $T_{\text {ship }}$ is the calendar life of the ship, and $N_{\text {use_days }}$ is the actual calendar life of the storage pack; it is represented as

$$
N_{\text {use_days }}=\frac{N_{\text {cycle }}}{N_{\text {daily }}},
$$

where $N_{\text {daily }}$ is the number of charge-discharge cycles in one day. It is defined as

$$
N_{\text {daily }}=\frac{E_{\text {ESS }}^{\text {exchanged }}}{\left(2 \cdot E_{n \mathrm{ESS}}^{\max } \cdot N_{\mathrm{ESS}}\right)},
$$

where $E_{\mathrm{ESS}}^{\text {exchanged }}$ is the total energy exchanged between the ESS and the ship power system in one day. It is defined as

$$
E_{\mathrm{ESS}}^{\text {exchanged }}=\sum_{t=1}^{T_{\text {day }}}\left(P_{\mathrm{ESSc}}(t)+P_{\mathrm{ESSd}}(t)\right) \Delta t,
$$

where $P_{\text {ESSc }}(t), P_{\text {ESSd }}(t)$ are charging, discharging rate of the ESS in period $t(\mathrm{~kW})$. Equations (14)-(17) indicate that the magnitude of $N_{\text {repESS }}$ is strictly related to the life cycle times of ESS, further related to the ESS charge/discharge strategy and its DOD in (6).

4.1.2. IPS Operation Cost. Operation cost $C_{M}$ of the IPS is calculated according to the optimal energy management problem. It comprises the operation cost of the DGs $C_{M_{-} \mathrm{DG}}$ and the ESSs $C_{M_{-} \text {ESS }}$.

$$
C_{M}=C_{M_{-} \mathrm{DG}}+C_{M_{-} \mathrm{ESS}} .
$$

The DGs' operation cost is the summary of every DG operation cost, which consists of the fuel consumption cost, the startup cost, the shutdown cost, and the maintenance cost, as shown in (19). Correspondingly, the mathematical models of the startup cost, shutdown cost, and maintenance cost are shown in (20)-(22).

$$
C_{M_{\mathrm{DG}}}=\sum_{i=1}^{N_{\mathrm{DG}}} \sum_{t=1}^{T_{\mathrm{day}}}\left(C_{i, \mathrm{DG}}^{\mathrm{fuel}}(t)+C_{i, \mathrm{DG}}^{\mathrm{stu}}(t)+C_{i, \mathrm{DG}}^{\mathrm{shd}}(t)+C_{i, \mathrm{DG}}^{\mathrm{O} \& M}(t)\right),
$$

$$
\begin{aligned}
C_{i, \mathrm{DG}}^{\mathrm{stu}}(t) & =c_{i, \mathrm{DG}}^{\mathrm{stu}} \delta_{i, \mathrm{DG}}^{\mathrm{stu}}(t), \\
C_{i, \mathrm{DG}}^{\mathrm{shd}}(t) & =c_{i, \mathrm{DG}}^{\text {shd }} \delta_{i, \mathrm{DG}}^{\text {shd }}(t), \\
C_{i, \mathrm{DG}}^{O \& M}(t) & =c_{i, \mathrm{DG}}^{\mathrm{O} \& M} P_{i, \mathrm{DG}}(t) .
\end{aligned}
$$

In (20), the startup cost $C_{i, \mathrm{DG}}^{\mathrm{stu}}(t)$ is defined as the product of a single time startup cost $c_{i, \mathrm{DG}}^{\text {stu }}$, which is a constant value, and the index value of startup action $\delta_{i, \mathrm{DG}}^{\mathrm{stu}}(t)$. The definition of shutdown cost is similar. In (22), the maintenance cost $C_{i, \mathrm{DG}}^{\mathrm{O \& M}}(t)$ is defined as the product of per unit power maintenance cost $c_{i, \mathrm{DG}}^{\mathrm{O} \& M}$ and the generator output power $P_{i, \mathrm{DG}}(t)$. 
In DG operation cost model of (20)-(22), the decision variables of the ith DG are the power output $P_{i, \mathrm{DG}}(t)$ and the operation status $\delta_{i, \mathrm{DG}}^{\text {onoff }}(t)$ of the ith DG in periods $t$.

The operation cost of ESSs is defined as the per unit changing power $c_{\mathrm{ESS}}^{o \& m}$ product the summary value of charging power $P_{\mathrm{ESSc}}(t)$ and discharging power $P_{\mathrm{ESSd}}(t)$.

$$
C_{M_{\text {EESS }}}=c_{\mathrm{ESS}}^{o \& m} \sum_{t=1}^{T_{\mathrm{day}}}\left(P_{\mathrm{ESSd}}(t)+P_{\mathrm{ESSC}}(t)\right) \Delta t .
$$

Equation (23) shows that, in ESS operation cost model, the decision variables of ESS are the charging power $P_{\text {ESSc }}(t)$ and discharging power $P_{\text {ESSd }}(t)$.

4.2. Constraints. Operation constraints of the shipboard IPS are presented as follows. It determines the energy management strategy and energy schedule of the ship power system.

4.2.1. Operation Constraints of the DGs. When the DGs run on, the operation of them must subject to the power output limitation, ramp up/down limitation, minimum runtime, and downtime limitation, as shown in (24)-(27), respectively.

$$
\begin{array}{r}
\delta_{i, \mathrm{DG}}^{\text {onoff }}(t) P_{i, \mathrm{DG}}^{\mathrm{min}} \leq P_{i, \mathrm{DG}}(t) \leq \delta_{i, \mathrm{DG}}^{\text {onoff }}(t) P_{i, \mathrm{DG}}^{\mathrm{max}}, \\
-R_{i, \mathrm{DG}}^{\mathrm{max}} \leq P_{i, \mathrm{DG}}(t)-P_{i, \mathrm{DG}}(t-1) \leq R_{i, \mathrm{DG}}^{\mathrm{max}}, \\
\delta_{i, \mathrm{DG}}^{\text {onoff }}(t)-\delta_{i, \mathrm{DG}}^{\text {onoff }}(t-1) \leq \delta_{i, \mathrm{DG}}^{\text {onoff }}\left(\tau_{i, \mathrm{DG}}^{\text {on }}\right), \\
\tau_{i, \mathrm{DG}}^{\text {on }}=t, \quad t+1, \ldots, \min \left(t+T_{i, \mathrm{DG}}^{\text {on }}-1, T_{\mathrm{day}}\right), \\
\delta_{i, \mathrm{DG}}^{\text {onoff }}(t-1)-\delta_{i, \mathrm{DG}}^{\text {onoff }}(t) \leq 1-\delta_{i, \mathrm{DG}}^{\text {onoff }}\left(\tau_{i, \mathrm{DG}}^{\text {off }}\right), \\
\tau_{i, \mathrm{DG}}^{\text {off }}=t, \quad t+1, \ldots, \min \left(t+T_{i, \mathrm{DG}}^{\text {off }}-1, T_{\mathrm{day}}\right), \\
\delta_{i, \mathrm{DG}}^{\text {stu }}(t)=\max \left(\delta_{i, \mathrm{DG}}^{\text {onoff }}(t)-\delta_{i, \mathrm{DG}}^{\text {onoff }}(t-1), 0\right), \\
\delta_{i, \mathrm{DG}}^{\text {shd }}(t)=\max \left(\delta_{i, \mathrm{DG}}^{\text {onoff }}(t-1)-\delta_{i, \mathrm{DG}}^{\text {onoff }}(t), 0\right),
\end{array}
$$

where $P_{i, \mathrm{DG}}^{\min }$ and $P_{i, \mathrm{DG}}^{\max }$ are the minimum and the maximum power output of the ith DG $(\mathrm{kW}), R_{i, \mathrm{DG}}^{\max }$ is the ramp up/down limit of the $i$ th DG $(\mathrm{kW}) . \tau_{i, \mathrm{DG}}^{\text {off }}$ and $\tau_{i, \mathrm{DG}}^{\mathrm{on}}$ are the introduced auxiliary parameters which are used to more efficiently express the limitation models of the minimum runtime and the minimum downtime. $T_{i, \mathrm{DG}}^{\text {on }}$ and $T_{i, \mathrm{DG}}^{\text {off }}$ are the minimum on and the minimum off time of the $i$ th DG $(h) . \delta_{i, \mathrm{DG}}^{\mathrm{stu}}(t)$ and $\delta_{i, \mathrm{DG}}^{\text {shd }}(t)$ are the introduced auxiliary binary variables to more efficiently express the $i$ th DG's startup cost and shutdown cost. In addition, $\delta_{i, \mathrm{DG}}^{\text {stu }}(t)$ and $\delta_{i, \mathrm{DG}}^{\text {shd }}(t)$ can be used to limit the operation times of $i$ th DG's startup and shutdown, if needed.

The operation problem with DGs' operation constraints can be expressed as a mixed integer quadratic programming model, which can be computed by some commercial software, such as ILOG, CPLEX, and GAMS. Of course, it can also be converted to a mixed linear quadratic programming model by piecewise linear approximation.

4.2.2. Operation Constraints of the ESSs. As is well-known, ESS's size determination is tightly coupled with the ESS's energy management strategy. Therefore, the modeling of optimal ESS sizing problems must consider the ESS's energy management strategy. A few literatures only set simple rulebased energy management strategy, which leads to the fact that the calculated ESS size is nonoptimal. Some other literatures divide the ESS sizing problem and the ESS energy management problem into two sub-problems; though the computation complexity is reduced, the computation burden is increased very large. In this paper, we integrate the ESS sizing problem and ESS energy management problem into a single model by introduce extra two constraints; however, the computation burden is not largely increased because no extra variable is introduced and it is still a mixed integer linear programming model. The detail description is shown as follows.

$$
\begin{gathered}
E_{n \mathrm{ESS}}^{\min } N_{\mathrm{ESS}} \leq E_{\mathrm{ESS}}(t) \leq E_{n \mathrm{ESS}}^{\max } N_{\mathrm{ESS}} \\
0 \leq P_{\mathrm{ESSc}}(t) \leq P_{\mathrm{ESSc}}^{\max } \\
0 \leq P_{\mathrm{ESSd}}(t) \leq P_{\mathrm{ESSd}}^{\max } \\
\delta_{\mathrm{ESSc}}(t) P_{\mathrm{ESSc}}^{\min } \leq P_{\mathrm{ESSc}}(t) \leq \delta_{\mathrm{ESSc}}(t) P_{\mathrm{ESSc}}^{\operatorname{mark}}, \\
\delta_{\mathrm{ESSd}}(t) P_{\mathrm{ESSd}}^{\min } \leq P_{\mathrm{ESSd}}(t) \leq \delta_{\mathrm{ESSd}}(t) P_{\mathrm{ESSd}}^{\operatorname{mark}} \\
\delta_{\mathrm{ESSc}}(t)+\delta_{\mathrm{ESSd}}(t) \leq 1 \\
E_{\mathrm{ESS}}(t+1)=E_{\mathrm{ESS}}(t)+\eta_{c} P_{\mathrm{ESSc}}(t) \Delta t-\left(\frac{P_{\mathrm{ESSd}}(t)}{\eta_{d} \Delta t}\right)-\varepsilon_{\mathrm{ESS}} N_{\mathrm{ESS}} \\
E_{\mathrm{ESS}}\left(T_{\mathrm{day}}+1\right)=E_{\mathrm{ESS}}(1) \\
P_{\mathrm{ESSc}}^{\max }=P_{n \mathrm{ESSc}}^{\max } N_{\mathrm{ESS}} \\
P_{\mathrm{ESSd}}^{\max }=P_{n \mathrm{ESSd}}^{\max } N_{\mathrm{ESS}} \\
P_{\mathrm{ESSc}}^{\operatorname{mark}}=P_{n \mathrm{ESSc}}^{\max } N_{\mathrm{ESS}}^{\max } \\
P_{\mathrm{ESSd}}^{\operatorname{mark}}=P_{n \mathrm{ESSd}}^{\max } N_{\mathrm{ESS}}^{\max }
\end{gathered}
$$

where $E_{n \mathrm{ESS}}^{\min }$ and $E_{n \mathrm{ESS}}^{\max }$ are the maximum and the minimum permit-able energy level of a single ESS module $(\mathrm{kWh})$, $\delta_{\mathrm{ESSc}}(t)$ and $\delta_{\mathrm{ESSd}}(t)$ are the introduced auxiliary binary variables to more efficiently express the charging and discharging status of the ESS in period $t$, and $\eta_{c}$ and $\eta_{d}$ are the charging and discharging efficiencies of ESS. $\varepsilon_{\mathrm{ESS}}$ is the selfloss of a single ESS module $(\mathrm{kWh} / \mathrm{h}) . T_{\text {day }}$ is the time interval numbers of one day. $P_{n \mathrm{ESSc}}^{\max }, P_{n \mathrm{ESSd}}^{\max }$ are the maximum charge and discharge power of a single ESS module $(\mathrm{kW}) . N_{\mathrm{ESS}}^{\max }$ is the maximum ESS modules can be used for this ship, and thus $P_{\mathrm{ESSc}}^{\mathrm{mark}}, P_{\mathrm{ESSd}}^{\mathrm{mark}}$ are the ESS maximum charge and discharge power for the ship. 
The storage capacity constraint is shown in (30), which indicates the actually decision variable of energy size is the storage modular numbers $N_{\text {ESS }}$. The storage power limitations are shown in (31)-(35), which consists of two parts, namely, the power magnitude limitation which is in (31), (32), and operation status limitation which is in (33)-(35). The storage dynamics model is shown in (36), which is the same as the other literatures. The energy consensus limitation is shown in (37), which indicates that at the beginning of one day the stored energy of the ESS should be the same. Equations (38), (39) are the formulation of the introduced auxiliary variables of $P_{\mathrm{ESSc}}^{\max }, P_{\mathrm{ESSd}}^{\max }, P_{\mathrm{ESSc}}^{\mathrm{mark}}$, and $P_{\mathrm{ESSd}}^{\mathrm{mark}}$.

The largest difference between the ESS model in this paper and that in other literatures is the description of ESS power limitation, as shown in (31)-(34). According to modeling experience in energy management problem, the ESS power and operation status limitations in ESS sizing problem will be expressed as in (40), (41), which constrain the ESS power limitations and its corresponding operation status limitations together and they should be expressed as a nonlinear programming model. In order to make the model in (40), (41) be efficiently resolved, it is divided into two subproblems to solve; the outer sub-problem determines the storage modular numbers $N_{\text {ESS }}$, and the inner sub-problem determines the corresponding optimal energy management scheme, namely, the optimal charge power $P_{\mathrm{ESSc}}(t)$ and discharge power $P_{\text {ESSd }}(t)$ in each period, as shown in Figure 2.

$$
\begin{aligned}
& \delta_{\mathrm{ESSc}}(t) P_{\mathrm{ESSc}}^{\mathrm{min}} \leq P_{\mathrm{ESSc}}(t) \leq \delta_{\mathrm{ESSc}}(t) P_{n \mathrm{ESSc}}^{\max } N_{\mathrm{ESS}} \\
& \delta_{\mathrm{ESSd}}(t) P_{\mathrm{ESSd}}^{\min } \leq P_{\mathrm{ESSd}}(t) \leq \delta_{\mathrm{ESSd}}(t) P_{n \mathrm{ESSc}}^{\max } N_{\mathrm{ESS}}
\end{aligned}
$$

In Figure 2, the outer optimization has to be implemented by intelligent optimization algorithms, such as genetic algorithm, particle swarm optimization, etc., which means the final result cannot be obtained immediately. In addition, the final result may be the nonoptimal result. The optimization model with (31)-(34) can optimize the storage modular numbers $N_{\text {ESS }}$ and the storage operation schedule simultaneously and can get the optimal result by analytical optimization algorithm.

4.2.3. Constraints of Power Balance and Spinning Reserve. In order to guarantee the safety of the shipboard power system, the power supply must be equal to the load demand all the time, where $P_{\text {load }}(t)$ represents the load demand of the ship in period $t(\mathrm{~kW})$.

$$
P_{\mathrm{ESSc}}(t)+P_{\text {load }}(t)=\sum_{i=1}^{N_{\mathrm{DG}}} P_{i, \mathrm{DG}}(t)+P_{\mathrm{ESSd}}(t)+P_{\mathrm{PV}}(t)
$$

In addition, the DGs must provide enough reserve capacity $R_{\mathrm{DG}}$ to meet the power and energy demand in emergency conditions.

$$
\sum_{i=1}^{N_{D G}} P_{i, \mathrm{DG}}^{\mathrm{max}} \cdot \delta_{i, \mathrm{DG}}^{\mathrm{onoff}}(t)+P_{\mathrm{PV}}(t)+P_{\mathrm{ESSd}}(t)-P_{\mathrm{ESSc}}(t) \geq R_{\mathrm{DG}} \cdot P_{\text {load }}(t)
$$

We should note that, because only a part of the ship rooftop can be applied to PV panels, the penetration level of $\mathrm{PV}$ generation is low and the impacts its randomness has can be neglected for a large power IPS ship.

4.2.4. Constraints of the ESS Module Numbers. Because the available space to deploy storage is limited, the storage number can be deployed and should be in a given range.

$$
N_{\text {ESS }}^{\min } \leq N_{\text {ESS }} \leq N_{\text {ESS }}^{\max }
$$

\section{Results and Discussion}

In this section, the proposed method is applied to a ferry ship equipped with low-voltage IPS. This ferry ship has two electric propulsion motors and four diesel generators. Moreover, it goes back and forth on the fixed shipping line every day. The sampled ship power data in one day is extracted from the onboard IAS and then averaged and presented in Figure 3, which is used to represent the typical data in ESS sizing optimization. It indicates that the load demand is fluctuant. The technical parameters of the generators are presented in Table 1 and the storage module data is presented in Table 2. The available ship rooftop is about $50 \mathrm{~m}^{2}$ and the PV generation of a typical spring day of the rooftop is shown in Figure 4. It indicates that the PV panels can supply power of $1400 \mathrm{kWh}$ in the daytime; this energy can effectively reduce the oil fuel consumption of the diesel generators.

For solving the optimization model in a short time, typical computational software such as MATLAB R2013a and ILOG's CPLEX v.12.0 is used in this paper. CPLEX v.12 optimization solver is utilized to solve the optimization model given in (18), and MATLAB 2013a and YALMIP toolbox are used for linking the CPLEX solver. All the computations are done on a PC with Intel(R) Core(TM) i5$3470,3.2 \mathrm{GHz}$ and $8 \mathrm{~GB}$ of RAM. The core of CPLEX optimization solver is brand-and-bound algorithm. Of course, the mathematical model can also be solved by heuristic or meta-heuristics approaches if the computation time has no restricted constraints. 


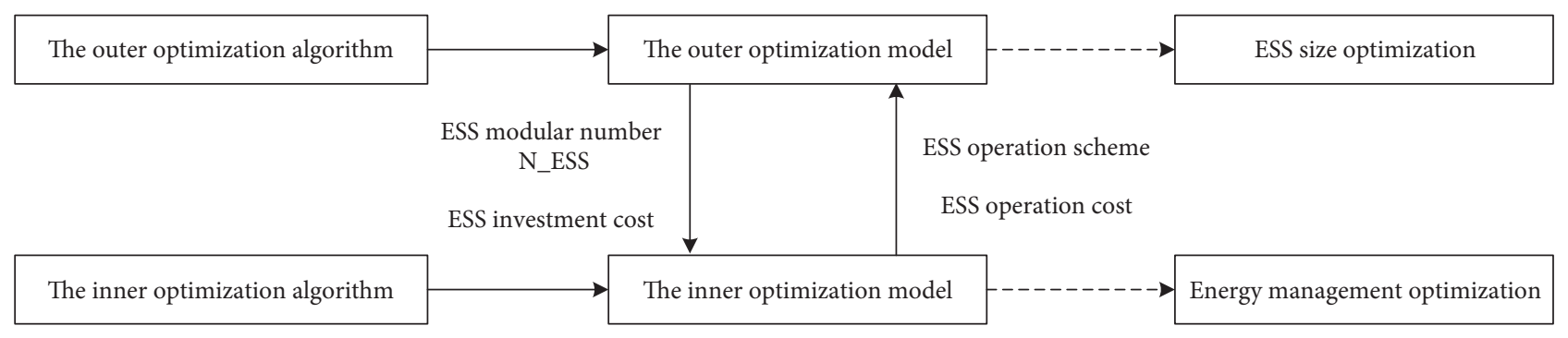

FIGURE 2: Bi-level configuration of traditional ESS optimal size problem with considering energy management.

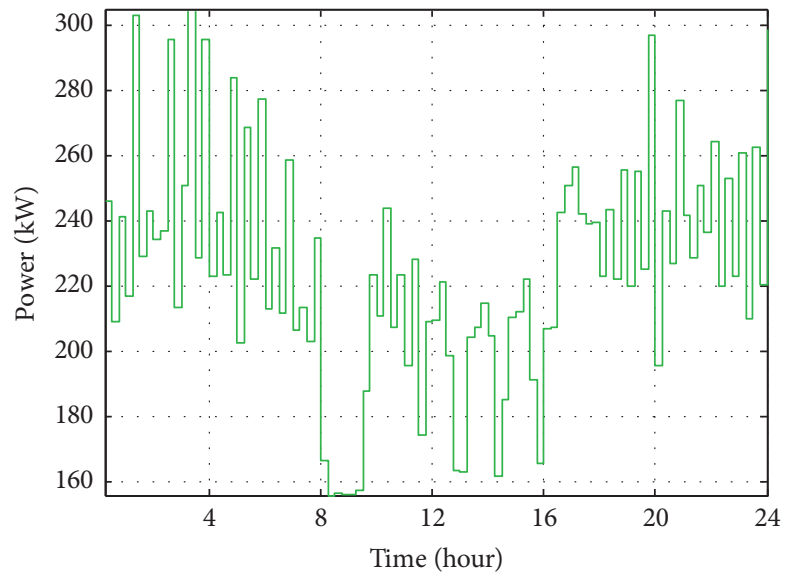

_ Ship power demand

FIGURE 3: Recorded load power.

TABle 1: Parameters of DGs.

\begin{tabular}{|c|c|c|c|c|c|}
\hline Num. & $P_{a}^{\min }(\mathrm{MW})$ & $P_{a}^{\max }(\mathrm{MW})$ & \multicolumn{2}{|c|}{$R_{a}^{\max }(\mathrm{MW} / \mathrm{min})$} & $T_{i, \mathrm{DG}}^{\mathrm{on}}(\mathrm{h})$ \\
\hline 1 & 0.1 & 5 & \multicolumn{2}{|c|}{4} & 1 \\
\hline 2 & 0.05 & 4 & \multicolumn{2}{|c|}{3.2} & 1 \\
\hline 3 & 0.05 & 4 & \multicolumn{2}{|c|}{3.5} & 1 \\
\hline 4 & 0.2 & 6 & \multicolumn{2}{|c|}{5} & 1 \\
\hline Type & $\alpha_{i, \mathrm{DG}}\left(\$ / \mathrm{MWh}^{2}\right)$ & $\beta_{i, \mathrm{DG}}(\$ / \mathrm{MWh})$ & $\gamma_{i, \mathrm{DG}}(\$ / \mathrm{h})$ & $c_{i, \mathrm{DG}}^{\mathrm{stu}}(\$)$ & $c_{i, \mathrm{DG}}^{\mathrm{shd}}(\$)$ \\
\hline 1 & 34.1 & 77 & 500 & 15 & 10 \\
\hline 2 & 55 & 28 & 510 & 15 & 10 \\
\hline 3 & 54 & 24 & 510 & 15 & 10 \\
\hline 4 & 32.6 & 84 & 490 & 16 & 10 \\
\hline Type & $a_{i, \mathrm{DG}}\left(\mathrm{kg} / \mathrm{kWh}^{2}\right)$ & $b_{i, \mathrm{DG}}(\mathrm{kg} / \mathrm{kWh})$ & $c_{i, \mathrm{DG}}(\mathrm{kg} / \mathrm{h})$ & $c_{a}^{o \& m}(\$ / \mathrm{MWh})$ & $T_{i, \mathrm{DG}}^{\mathrm{off}}(\mathrm{h})$ \\
\hline 1 & 0.0041 & -5.55 & 14.09 & 1.4 & 1 \\
\hline 2 & 5.64 & -6.05 & 12.54 & 1 & 1 \\
\hline 3 & 5.62 & -6.06 & 12.55 & 1 & 1 \\
\hline 4 & 3.38 & -3.55 & 15.33 & 1.6 & 1 \\
\hline
\end{tabular}

TABLE 2: Parameters of a storage module.

Nominal capacity

DoD

Max. charging/discharging rate

Life cycle

Charging/discharging efficiency

Capital cost of storage module

Capital cost of converter

Operation and maintenance cost
$105 \mathrm{Ah} / 51.2 \mathrm{~V}$ $70 \%$

$0.5 \mathrm{C} / 2 \mathrm{C}$

3000 cycles

$0.95 / 0.95$

$200 \$ / \mathrm{kWh}$

$100 \$ / \mathrm{kW}$

$0.01 \$ / \mathrm{kWh}$
Besides, the time interval of energy management is $1 \mathrm{~min}$, and the minimum spinning reserve ratio of the DGs is set to $5 \%$.

5.1. Sizing of ESSs. By implementing the proposed ESS sizing model, the operation routines of the DGs and the ESS device are presented in Figures 5-7. The total solving time of the optimization model is $12.44 \mathrm{~s}$, which is much shorter than 


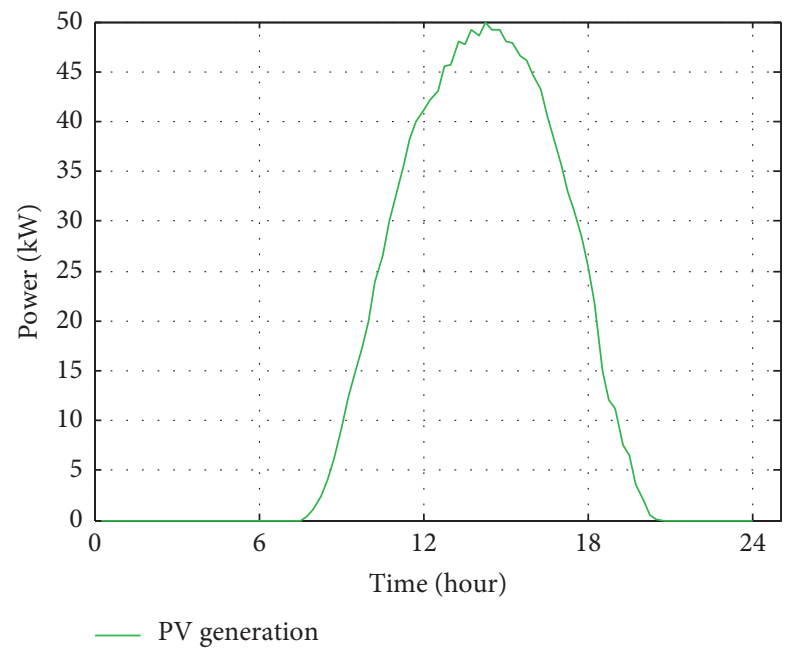

Figure 4: PV generation of a typical day.

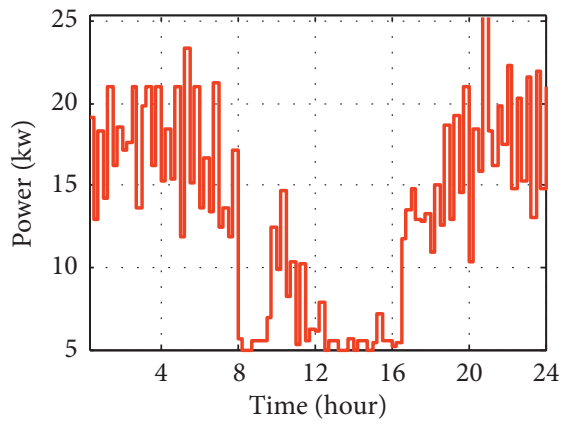

- DG1 power

(a)

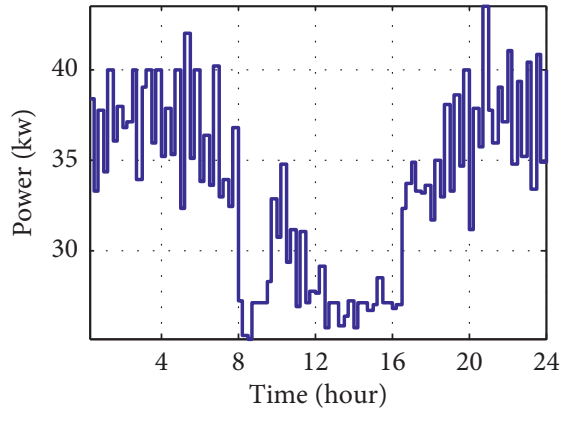

— DG2 power

(b)

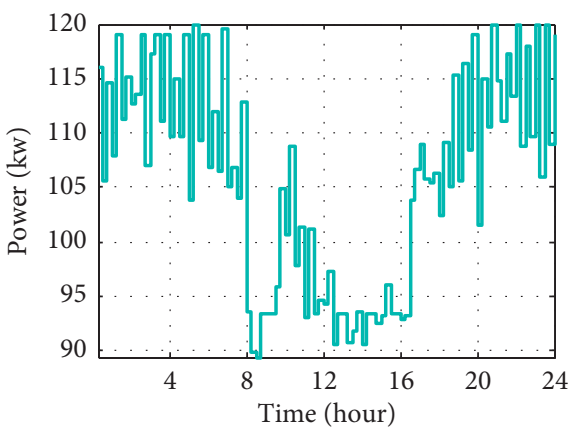

— DG4 power

(d)

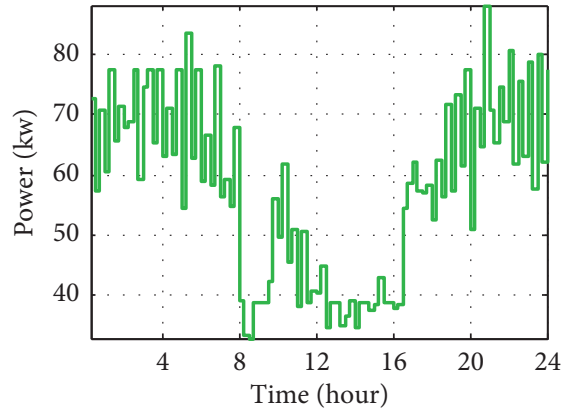

— DG3 power

(c)

Figure 5: Operation schedule of DGs with ESS.

the two-layer optimization method in [21]. In [21], the total optimization time probably needs several hours because the time-consuming energy management optimization model has to be iterated hundreds of times.

By observing Figures 5 and 6 , it can be found that the ESS plays an important role in the IPS operation. When the ship has no ESS, the DGs power outputs are $1328 \mathrm{kWh}$, $3312 \mathrm{kWh}, 5536 \mathrm{kWh}$, and $10062 \mathrm{kWh}$, respectively. Meanwhile, when the ship has ESS integration, the DGs power outputs are $1261 \mathrm{kWh}, 3208 \mathrm{kWh}, 5543 \mathrm{kWh}$, and $10165 \mathrm{kWh}$, respectively, as shown in Table 3. The operation cost of the DGs in one day is reduced $50 \$$ with the integration of the ESS device.

The ESS device in Figure 7 is mainly used to improve the fuel efficiency of the DGs because the load demand is fluctuant. There are 464 battery modules which are needed for this ferry ship, the rated ESS capacity is $155 \mathrm{kWh}$, and the investment cost of this ESS device is 


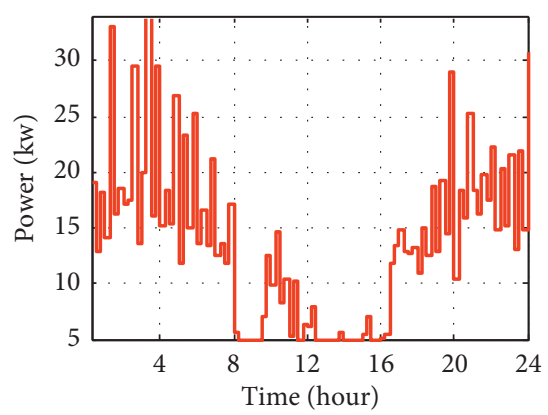

— DG1 power

(a)

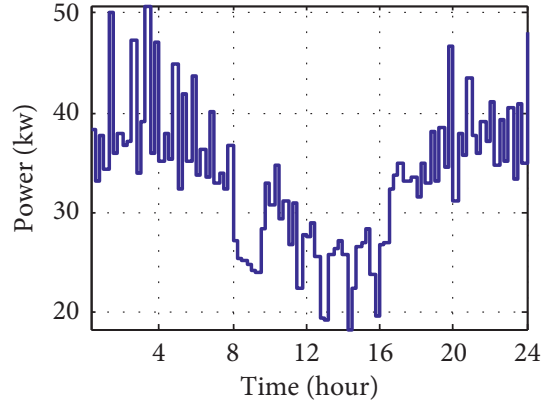

— DG2 power

(b)

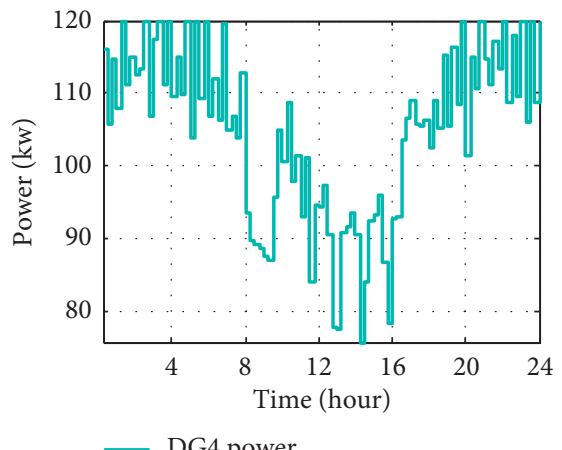

(d)

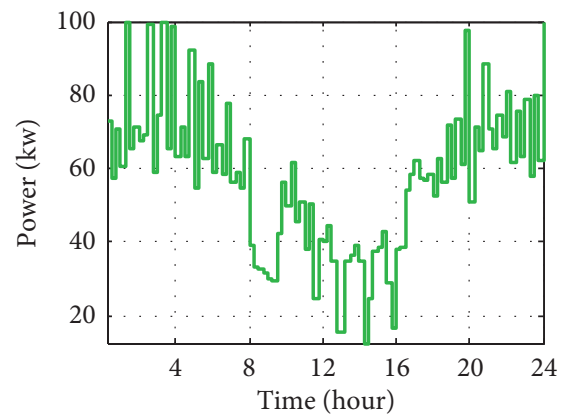

FIgURE 6: Operation schedule of DGs without ESS.

TABle 3: Power outputs of DGs with/without ESS.

DGs number

Power outputs without ESS (kWh)

Power outputs with ESS (kWh)

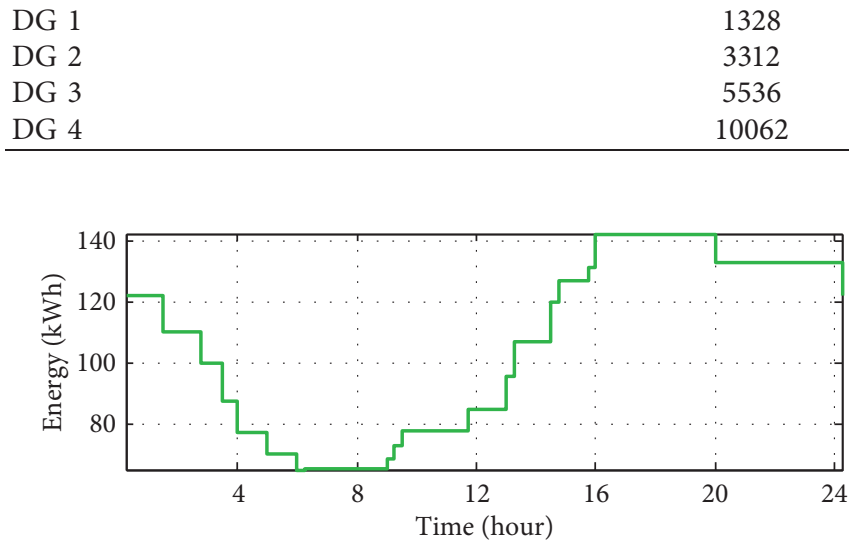

_ ESS energy schedule

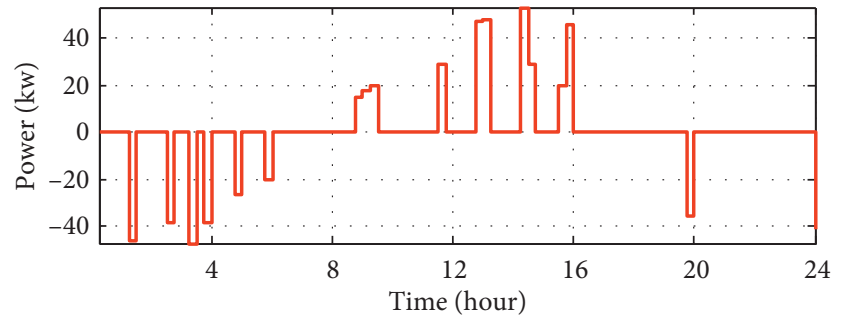

_ ESS power schedule

(a)

(b)

FIgURE 7: Operation schedule of ESSs. 
46500\$, and the ESS device does not need to be replaced over the ship life. If this ferry ship can work 300 days in one year, only need 3 years can recover the initial investment cost.

Meanwhile, the one-day $\mathrm{CO}_{2}$ emission of the DGs with or without ESS is $4439.1 \mathrm{~kg}$ and $4474.6 \mathrm{~kg}$ in one day, as shown in Table 4, respectively. Table 4 indicates that the ship can reduce $\mathrm{CO}_{2}$ emission $259.2 \mathrm{t}$ over its whole life. Therefore, the advantage of using ESS is significant no matter whether from the economic view or the carbon emission-reducing view.

5.2. Sensitivity Studies. Generally, the ESS capacity size results will be different with different ESS parameters. In this paper, the impacts of storage charging/discharging rate, the ESS module cost, and the ESS cycle life cycle are discussed.

5.2.1. Impacts of Discharging Rate. As is known to all, ESS discharge/charge rate is an important parameter to evaluate the performance of ESS. The larger the discharge/charge rate, the better the dynamic response performance, and it needs less ESS capacity to deal with the same power randomness. In addition, the charge rate is usually smaller than the discharge rate for a battery.

In order to investigate the relationship between the discharging rate and the ESS sizing optimization, the ESS discharging rate varies from $0.4 \mathrm{C}$ to $4 \mathrm{C}$ and the charging rate varies from $0.1 \mathrm{C}$ to $1 \mathrm{C}$ simultaneously, where $\mathrm{C}$ is used to represent the rate of the discharge/charge current for ESS and $1 \mathrm{C}$ indicates the ESS can be fully discharged/charged in one hour. The discharge rate increment is $0.4 \mathrm{C}$, and the charge rate increment is $0.1 \mathrm{C}$. The results of every time are shown in Figure 8.

Figure 8 indicates that when the ESS charge rate is very low, the charge rate increases can make the ESS more economical and it has significant impacts on the ESS size; however, if the charge rate increases further, its impacts on the ESS economy are neglected. The charge rate and discharge rate of the battery optimized in this paper are both $0.4 \mathrm{C}$. In order to reduce the initial investment cost of the ESS, the battery charge rate must be higher than $0.4 \mathrm{C}$.

5.2.2. Impacts of ESS Cycle Life. Similarly, the ESS cycle life is another important index to evaluate the performance of ESS. The longer the cycle life is, the lower the average operation and investment costs are.

In order to investigate the relationship between the cycle life and the ESS sizing optimization, the cycle life varies from 500 times to 4000 times. The increment of each time is set as 500. The simulation results are presented in Figure 9.

In Figure 9, the longer the cycle life of the battery is, the more battery unit will be applied. If the cycle life of the battery is too short, the ESS investment cost cannot be recovered. Therefore, as the battery technology improves, more and more ships have the idea to apply ESS.
TABLE 4: $\mathrm{CO}_{2}$ emission of DGs with/without ESS.

\begin{tabular}{lcc}
\hline $\mathrm{CO}_{2}$ emissions & $\begin{array}{c}\text { Without ESS } \\
(\mathrm{kg})\end{array}$ & $\begin{array}{c}\text { With ESS } \\
(\mathrm{kg})\end{array}$ \\
\hline In one day & 4474.6 & 4439.1 \\
In one year & $1.663 * 10^{6}$ & $1.62 * 10^{6}$ \\
$\begin{array}{l}\text { Over the ferry's whole life }(20 \\
\text { years })\end{array}$ & $3.27 * 10^{7}$ & $3.24 * 10^{7}$ \\
\hline
\end{tabular}

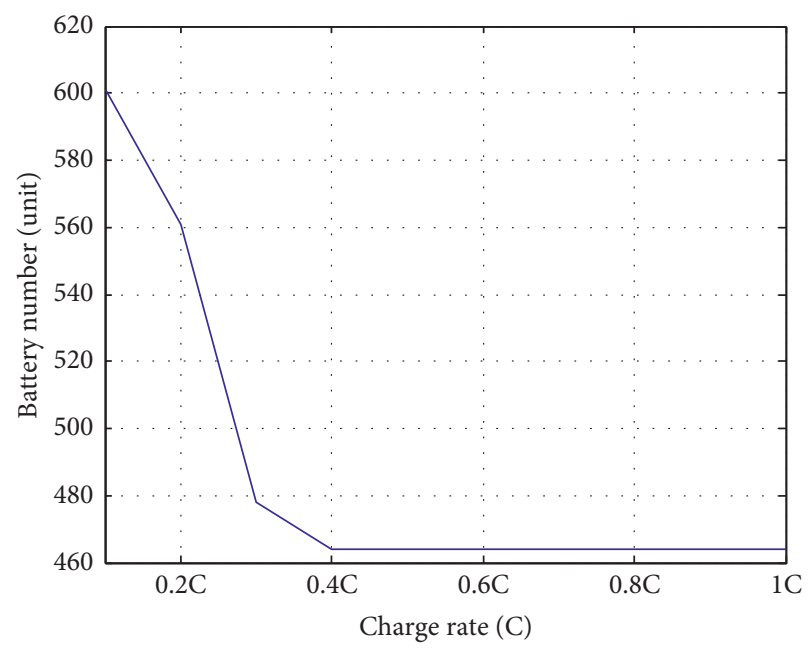

FIGURE 8: Relationship between the battery number and charge rate.

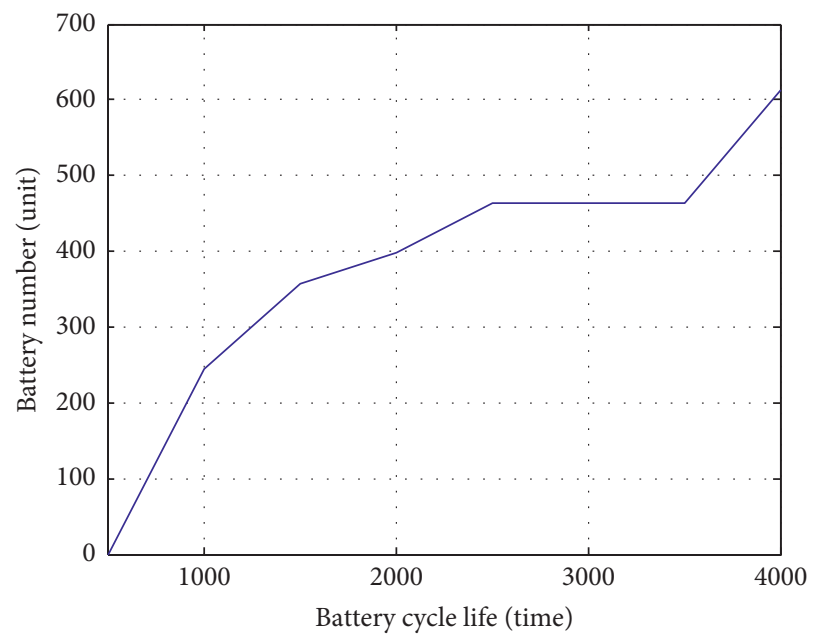

FIgURE 9: Relationship between the battery number and cycle life.

5.2.3. Impacts of ESS Investment Cost. In order to investigate the relationship between the ESS investment cost and the ESS sizing optimization, the storage pack investment cost decreases from $200 \$ / \mathrm{kWh}$ a module to $100 \$ / \mathrm{kWh}$. The decrement each time is set as $20 \$$. The converter investment cost will also be decreased correspondingly. The results are shown in Figure 10.

Figure 10 indicates that the ESS optimal size is very sensitive to the storage unit investment cost, and the battery unit number increase speed is faster than the battery cost decrease cost. 


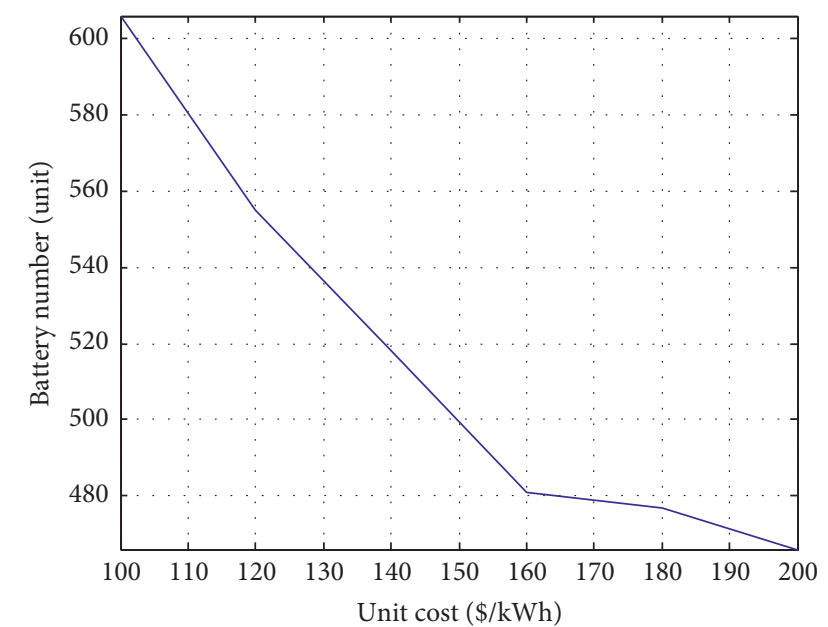

FIgURE 10: Relationship between the battery number and energy cost.

\section{Conclusions}

In this work, an optimal energy management strategy based on ESS sizing selection for a shipboard power system has been presented. This method has been developed in a flexible and general way to apply it to different types of shipboard power systems and different energy storage technologies. The effectiveness of the proposed method has been validated through a low-voltage integrated power system-based ferry ship. The case study shows that the solving time of the model proposed in this paper can be significantly reduced while comparing with the conventional two-stage modeling method. Besides, the whole life operation cost of the ferry ship can be reduced by $2.3 \%$ and the $\mathrm{CO}_{2}$ emission reduction can be reduced by $5.52 \%$ by integrating ESS. The impacts of charging/discharging rate, storage module investment cost, and ESS cycle life are all investigated to help the selection of ESS in the future.

Future studies will be developed to investigate the sizing optimization problem of hybrid energy storage systems by considering more complex and more efficient models, the optimization of the depth of discharge, and the constraints of space and volume. Besides, renewable energy resources, such as PV generations and wind turbines, will be considered in the further model.

\section{Data Availability}

The data used to support the findings of this study are available from the corresponding author upon request.

\section{Conflicts of Interest}

The authors declare that there are no conflicts of interest regarding the publication of this paper.

\section{Acknowledgments}

This work was partially supported by the Foundation for the National Key Laboratory of Science and Technology under grant nos. 614221720190506 and 614221720190507.

\section{References}

[1] UNCTAD, Review of Maritime Transport 2015, UNCTAD, Geneva, Switzerland, 2015, http://unctad.org/webflyer/ review-martime-transport-2015.

[2] IMO, "Revised maprol annex VI: regulations for the prevention of air pollution from ships"," 2015, http://www.imo. org/\%20OurWork/Environment/Pages/Air-Pollution.aspx.

[3] A. Serpi, M. Porru, and A. Damiano, "A novel highly integrated hybrid energy storage system for electric propulsion and smart grid applications," Advancements in Energy Storage Technologies, vol. 71, pp. 79-101, 2018.

[4] Z. Jin, L. Meng, and J. M. Guerrero, "Hierarchical control design for a shipboard power system with DC distribution and energy storage aboard future more-electric ships," IEEE Transaction on Industrial Informatics, vol. 14, no. 2, pp. 703-714, 2017.

[5] J. S. Thongam, M. Tarbouchi, A. F. Okou, D. Bouchard, and R. Beguenane, "All-electric ships-a review of the present state of the art," in Proceedings of the 2013 English International Conference and Exhibition on Ecological Vehicles and Renewable Energies (EVER), pp. 1-8, Monte Carlo, Monaco, March 2013.

[6] N. Doerry, J. Amy, and C. Krolick, "History and the status of electric ship propulsion, integrated power systems, and future trends in the U.S. navy," Proceedings of the IEEE, vol. 103, no. 12, pp. 2243-2251, 2015.

[7] Y. Zhang, C. Yuan, and H. Li, "A optimization method used for sailing route of solar ship," in Proceedings of the 2017 4th International Conference on Transportation Information and Safety(ICTIS), pp. 541-544, Banff, Canada, August 2017.

[8] F. D. Kanellos, G. J. Tsekouras, and N. D. Hatziargyriou, "Optimal demand-side management and power generation scheduling in an all-electric ship," IEEE Transactions on Sustainable Energy, vol. 5, no. 4, pp. 1166-1175, 2014.

[9] K. Yigit and B. Acarkan, "A new electrical energy management approach for ships using mixed energy sources to ensure sustainable port cities," Sustainable Cities and Society, vol. 40, pp. 126-135, 2018.

[10] G. Hug and A. Kargarian, "Optimal sizing of energy storage systems: a combination of hourly and intra-hour time perspectives," IET Generation Transmission \& Distribution, vol. 10, no. 3, pp. 594-600, 2016.

[11] N. Devillers, M. C. Péra, D. Bienaimé, and M. L. Grojo, "Influence of the energy management on the sizing of electrical energy storage systems in an aircraft," Journal of Power Sources, vol. 270, pp. 391-402, 2014.

[12] Z. Jin, G. Sulligoi, R. Cuzner, L. Meng, J. C. Vasquez, and J. M. Guerrero, "Next-generation shipboard dc power system: introduction smart grid and dc microgrid technologies into maritime electrical networks," IEEE Electrification Magazine, vol. 4, no. 2, pp. 45-57, 2016.

[13] M. D. A. Al-Falahi, K. S. Nimma, S. D. G. Jayasinghe, H. Enshaei, and J. M. Guerrero, "Power management optimization of hybrid power systems in electric ferries," Energy Conversion and Management, vol. 172, pp. 50-66, 2018.

[14] A. Maleki and F. Pourfayaz, "Sizing of stand-alone photovoltaic/wind/ diesel system with battery and fuel cell storage devices by harmony search algorithm," Journal of Energy Storage, vol. 2, pp. 30-42, 2015.

[15] R. D. Geertsma, R. R. Negenborn, K. Visser, and J. J. Hopman, "Design and control of hybrid power and propulsion systems for smart ships: a review of developments," Applied Energy, vol. 194, pp. 30-54, 2017. 
[16] J. Xiao, L. Bai, F. Li, H. Liang, and C. Wang, "Sizing of energy storage and diesel generators in an isolated microgrid using discrete fourier transform (DFT)," IEEE Transactions on Sustainable Energy, vol. 5, no. 3, pp. 907-916, 2014.

[17] S. Wen, H. Lan, D. C. Yu et al., "Optimal sizing of hybrid energy storage sub-systems in PV/diesel ship power system using frequency analysis," Energy, vol. 140, pp. 198-208, 2017.

[18] Y. Liu, X. Wu, J. Du, Z. Song, and G. Wu, "Optimal sizing of a wind-energy storage system considering battery life," Renewable Energy, vol. 147, pp. 2470-2483, 2020.

[19] R. Wang, R. C. Purshouse, and P. J. Fleming, "Preferenceinspired co-evolutionary algorithms for many-objective optimisation," IEEE Transactions on Evolutionary Computation, vol. 17, no. 4, pp. 474-494, 2013.

[20] K. W. Li, T. Zhang, and R. Wang, "Deep reinforcement learning for multi-objective optimization," IEEE Transaction on Cybernetics, vol. 51, no. 6, pp. 3103-3114, 2020.

[21] C. Yan, G. K. Venayagamoorthy, and K. A. Corzine, "Optimal location and sizing of energy storage modules for a smart electric ship power system," in Proceedings of the 2011 IEEE SSCI Symposium on Computational Intelligence Application in Smart Grid (CIASG 2011), pp. 1-8, Paris, French Guiana, April 2011.

[22] S. Mashayekh, Z. Wang, L. Qi, J. Lindtjorn, and T. A. Myklebust, "Optimum sizing of energy storage for an electric ferry ship," in Proceedings of the 2012 IEEE Power and Energy Society General Meeting (PESGM 2012), pp. 1-8, San Diego, CA, USA, July 2012.

[23] A. Boveri, F. Silvestro, and M. Molinas, "Optimal sizing of energy storage systems for shipboard applications," IEEE Transaction on Energy Conversion, vol. 34, no. 2, pp. 801-811, 2018.

[24] R. Wang, Z. Zhou, H. Ishibuchi, T. Liao, and T. Zhang, "Localized weighted sum method for many-objective optimization," IEEE Transactions on Evolutionary Computation, vol. 22, no. 1, pp. 3-18, 2018.

[25] R. Wang, Q. Zhang, and T. Zhang, "Decomposition based algorithms using pareto adaptive scalarizing methods," IEEE Transactions on Evolutionary Computation, vol. 20, no. 6, pp. 821-837, 2016.

[26] M. Othman, A. Anvari-Moghaddam, and N. Ahamad, "Scheduling of power generation in hybrid shipboard microgrids with energy storage systems," in Proceedings of the 2018 IEEE International Conference on Environment and Electrical Engineering and 2018 IEEE Industrial and Commercial Power System Europe (EEEIC/I\&CPS Europe), Palermo, Italy, June 2018.

[27] J. Li, X. Zheng, and X. Ai, "Optimal design of capacity of distributed generation in island standalone microgrid," Transaction of China Electrotechnical Society, vol. 31, no. 10, pp. 176-184, 2016.

[28] S. Y. Kim, S. Choe, S. Ko, and S. K. Sul, "A naval integrated power system with a battery energy storage system," IEEE Electrification Magazine, vol. 3, no. 2, pp. 22-33, 2015.

[29] Z. C. Shi, R. Wang, T. Zhang et al., "Optimal design of gridconnected hybrid renewable energy systems using multiobjective evolutionary algorithm," Scientia Iranica, vol. 24, no. 6, pp. 3418-3156, 2017.

[30] B. Lefevre, B. Herteleer, S. D. Breucker, and J. Driesen, "Bayesian inference based MPPT for dynamic irradiance conditions," Solar Energy, vol. 174, pp. 1153-1162, 2018.

[31] M. Cao, Q. Xu, and X. Qin, "Battery energy storage sizing based on a model predictive control strategy with operational constraints to smooth the wind power," Electrical Power \& Energy System, vol. 115, pp. 105-111, 2020.

[32] L. Farrier and R. Bucknall, "Assessing battery energy storage for integration with hybrid propulsion and high energy weapons," in Proceedings of the Engine As A Weapon International Symposium VIII(EAAW VIII), London, UK, July 2019.

[33] O. Tremblay, L. Dessaint, and A. Dekkiche, "A generic battery model for the dynamic simulation of hybrid electric vehicles," in Proceedings of the 2017 IEEE Vehicle Power and Propulsion Conference(VPPC 2007), pp. 284-289, Arlington, TX, USA, September 2007.

[34] J. Manwell, A. Rogers, G. Hayman, C. Avelar, and J. McGowan, "Hybrid2-a hybrid system simulation model: theory manual," Tech. Rep. Subcontract No. XL-1-11126-1-1, National Renewable Energy Laboratory, Univ. Massachusetts, Boston, MA, USA, 2006.

[35] C. R. Lashway, A. T. Elsayed, and O. A. Mohammed, "Hybrid energy storage management in ship power systems with multiple pulsed loads," Electric Power Systems Research, vol. 141, pp. 50-62, 2016. 